\title{
Pseudoaneurysm of Mitral-Aortic Intervalvular Fibrosa
}

\author{
Tahir Tak, MD, PhD, Department of Cardiology, Marshfield Clinic, Marshfield, WI 54449
}

\begin{abstract}
Pseudoaneurysm of the mitral-aortic intervalvular fibrosa (MAIVF) is one of the rare complications of infective endocarditis. Echocardiography plays an important role in the diagnosis of this condition. Transesophageal echocardiography (TEE) is generally superior to the transthoracic approach in the evaluation of the complications resulting from infective endocarditis. In this report, we discuss a case of infective endocarditis complicated by the development of a pseudoaneurysm of the MAIVF. The anatomic relationship of structures contiguous to the MAIVF and the salient echocardiographic findings of this clinical condition are presented. At surgery the diagnosis was confirmed and appropriate treatment instituted. The postoperative course was uncomplicated.
\end{abstract}

\section{INTRODUCTION}

Transesophageal echocardiography (TEE) is known to be superior to transthoracic echocardiography (TTE) in the visualization of valvular vegetations, abscesses and other complications in patients with infective endocarditis. ${ }^{1}$ One such complication is the development of false aneurysms of the mitral-aortic intervalvular fibrosis. ${ }^{2-3}$ These false aneurysms are prone to rupture, embolize or even cause further destruction of the aortic or mitral valve apparatus. ${ }^{4-5}$ It is, therefore, important to recognize this complication early, and to institute appropriate surgical treatment in a timely fashion in order to decrease the morbidity and mortality. This case report describes a patient with a protracted course of infective endocarditis who was diagnosed with a false aneurysm of the mitral-aortic intervalvular fibrosa (MAIVF). Since TTE was unable to provide adequate information, TEE was performed and proved extremely valuable in the proper diagnosis and treatment of our patient. This case report was approved by the Institutional Review Board.

REPRINT REQUESTS:

Tahir Tak, MD

Department of Cardiology

Marshfield Clinic

1000 North Oak Avenue

Marshfield, WI 54449

Telephone: 715-387-5301

Fax: 715-389-4555

Email: tak.tahir@marshfieldclinic.org
KEYWORDS:

Infective endocarditis; Aortic valve; Intervalvular fibrosa 


\section{CASE REPORT}

A 56-year-old female with a history of stage 0 chronic lymphocytic leukemia (CLL) was admitted for chest pain, general fatigue and an abnormal echocardiogram suggesting infective endocarditis. She had undergone coronary artery bypass graft $(\mathrm{CABG})$ and prosthetic aortic valve replacement approximately 4 months earlier. About 2 weeks prior to admission, her primary care physician had treated her with antibiotics for low-grade fever. Blood cultures ordered on an outpatient basis revealed group D Streptococcus. She was subsequently admitted for intravenous antibiotic treatment. On physical examination, her blood pressure was $135 / 80 \mathrm{~mm} \mathrm{Hg}$, and her pulse was 80. Examination of the lungs and heart revealed mild inspiratory crackles, grade $2 / 6$ systolic murmur at the second intercostal space, and no gallops. Abdominal exam was within normal limits. No skin lesions were noted and the extremities did not show any peripheral edema. A two dimensional TTE revealed some 'shagginess' around the left sinus of Valsalva. There was no gradient across the bioprosthetic valve. Subsequent TEE showed a large pseudoaneurysm in the region of the MAIVF. There was systolic expansion within the area of the MAIVF with color flow evidence of communication with the left ven- tricular outflow tract (figure 1). Mild aortic regurgitation was noted by color flow imaging. The bioprosthetic aortic valve appeared to be functioning well. The basal inferior wall was akinetic. Global left ventricular function was within normal limits. There was no pericardial effusion. The blood chemistries were within normal limits except for the hemoglobin, hematocrit and white blood count which, were $10.5 \%$, $32.4 \%$ and 12,000 , respectively. A chest x-ray showed a normal cardiac silhouette and no evidence of pulmonary vascular congestion. There was evidence of prosthetic aortic valve replacement. No infiltrates were seen.

At surgery, an abscess was seen involving the area of the left and non-coronary sinuses with development of a pseudoaneurysm of the MAIVF and communication with the left ventricular outflow tract as seen by echocardiography. This area was thoroughly débrided to remove the necrotic tissue and infiltrated with antibiotics. A pericardial patch was used to repair the fistula connecting the left ventricular outflow tract with the MAIVF. The original prosthetic valve was excised and replaced with a Carbomedics No. 23 aortic valve. The remainder of the hospital course was uncomplicated.
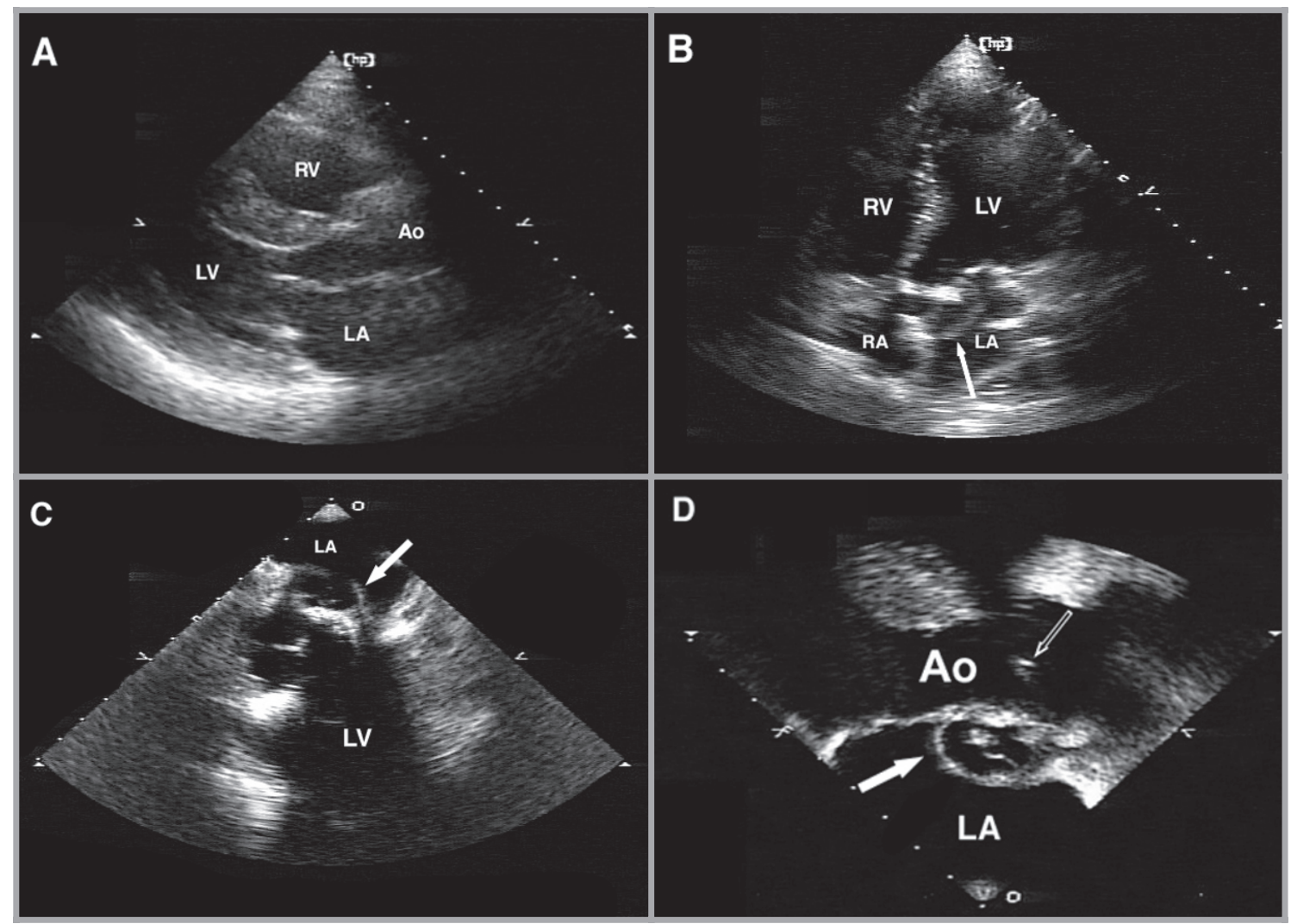

Figure 1.

A and B: Transthoracic parasternal and apical two chamber views showing the pseudoaneurysm and its proximity to the prosthetic aortic valve (see arrow).

C and D: Transesophageal echocardiography delineating the pseudoaneurysm and encroachment of the MAIVF (indicated by bold arrow). The aortic valve is indicated by the open arrow. MAIVF=mitral-aortic intervalvular fibrosa. 


\section{DISCUSSION}

Prosthetic valve endocarditis (PVE) has been reported in up to $10 \%$ of patients during the lifetime of the prosthesis. For mechanical prosthesis, the incidence is highest in the first few weeks after surgery and declines substantially thereafter. Early PVE (less than 2 months post valve implantation) results from valve contamination during the perioperative period and is usually caused by Staphylococcus epidermidis and S. aureus. Late PVE (more than 2 months post valve implantation) is most commonly caused by $S$. viridans and other microorganisms that closely resemble those of native valve endocarditis. ${ }^{6}$ Some investigators believe that many cases of PVE that occur during the first year after surgery are acquired at the time of implantation and further recommend that the time limit for early disease be extended to 6 months or even 1 year from implantation. ${ }^{7}$ The prognosis in PVE is clearly worse than in native valve. Late PVE has a mortality rate of $19 \%$ to $50 \%$ which is slightly better than that of early PVE which carries a mortality rate of $41 \%$ to $80 \%{ }^{7}$

The case is that of infective endocarditis complicated by pseudoaneurysm of the MAIVF. MAIVF is the junction between the left half of the non-coronary cusp and the adjacent third of the left coronary cusp of the aortic valve and the anterior mitral leaflet. Previous reports have described the pathoanatomic and angiographic features of pseudoaneurysms of the left ventricular outflow tract due to perforation of the MAIVF. Perforation or rupture of this area is usually secondary to blunt chest trauma or as a result of infection. ${ }^{4}$ TTE may show the aneurysm located behind the aortic root by the occurrence of systolic expansion and diastolic collapse seen by echocardiography. Color flow imaging helps, especially in confirming this phenomenon. However, TEE is a more sensitive technique to evaluate this condition, since the details are likely to be seen well. ${ }^{2}$ This should be differentiated from a typical aortic ring abscess which does not show this phenomenon. The extension of the infectious process is thought to be either due to the contiguous spread or due to the aortic regurgitant jet striking the subvalvular structures. Once a pseudoaneurysm has developed, it is prone to rupture, resulting either in pericardial tamponade, an eccentric jet of mitral regurgitation, severe regurgitation, or in a fistula connecting the left ventricle to the ascending aorta. Smaller pseudoaneurysms may be more difficult to distinguish from an abscess, but the systolic expansion and diastolic collapse of the area should alert the echocardiographer to this condition and help in differentiating between these two important clinical entities. ${ }^{5,8}$

MAIVF is a relatively avascular structure and, as such, it offers little resistance to infection. A general necropsy image (not from our patient) is shown in figure 2, and shows the anatomical details of the areas around the MAIVF. Infection of this area and of other subaortic structures like the anterior mitral leaflet may be more common than is commonly appreciated. It is, therefore, essential that echocardiographers focus their attention to the subaortic area and to the anterior mitral leaflet in all patients with infective endocarditis. A TTE should be done initially, but if there is any doubt regarding the thickening at the posterior aortic root or at the base of the anterior mitral leaflet, TEE should be utilized to further evaluate this area as it has a much higher sensitivity and specificity in diagnosing such complications. 8,9

In summary, pseudoaneurysm of the MAIVF is a relatively rare complication of infective endocarditis. It should be kept in mind in patients who are suspected of an abscess in the region of the sinus of Valsalva. TEE is more sensitive in accurately predicting this complication and the threshold for performing this test in such patients should be low.

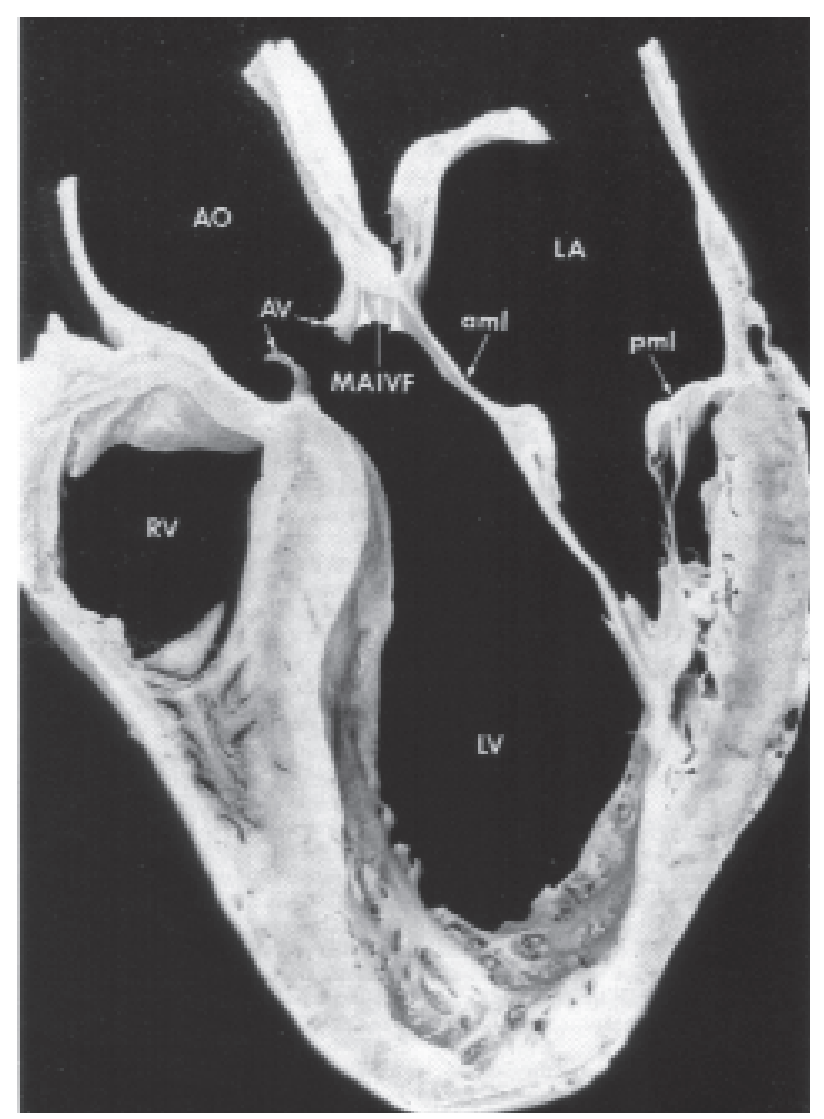

Figure 2. General necropsy image of the heart (not from our patient) showing the relation of the aortic valve (AV), annulus and root (AO) to the subaortic structures, including the MAIVF. The anterior mitral leaflet becomes continuous with the posterior aortic root.

MAIVF=mitral-aortic intervalvular fibrosa aml=anterior mitral leaflet $\mathrm{pml}=$ posterior mitral leaflet $L V=$ left ventricular $\mathrm{RV}=$ right ventricular $\mathrm{LA}=$ left atrium 


\section{REFERENCES}

1. Shively BK, Gurule FT, Roldan CA, Leggett JH, Schiller NB. Diagnostic value of transesophageal compared with transthoracic echocardiography in infective endocarditis. J Am Coll Cardiol 1991;18:391-397.

2. Karalis DG, Bansal RC, Hauck AJ, Ross JJ Jr, Applegate PM, Jutzy KR, et al. Transesophageal echocardiographic recognition of subaortic complications in aortic valve endocarditis. Clinical and surgical implications. Circulation 1992;86:353-362.

3. Agirbasli M, Fadel BM. Pseudoaneurysm of the mitral-aortic intervalvular fibrosa: a long-term complication of infective endocarditis. Echocardiography 1999;16:253-257.

4. Chesler E, Korns ME, Porter GE, Reyes CN, Edwards JE. False aneurysm of the left ventricle secondary to bacterial endocarditis with perforation of the mitral-aortic intervalvular fibrosa. Circulation 1968;37:518-523.

5. Afridi I, Apostolidou MA, Saad RM, Zoghbi WA. Pseudoaneurysms of the mitral-aortic intervalvular fibrosa: dynamic characterization using transesophageal echocardiographic and Doppler techniques. J Am Coll Cardiol 1995;25:137-145.

6. Rutledge R, Kim BJ, Applebaum RE. Actuarial analysis of the risk of prosthetic valve endocarditis in 1,598 patients with mechanical and bioprosthetic valves. Arch Surg 1985;120:469-472.

7. Cowgill LD, Addonizio VP, Hopeman AR, Harken AH. Prosthetic valve endocarditis. Curr Probl Cardiol 1986;11:617-664.

8. Bansal RC, Graham BM, Jutzy KR, Shakudo M, Shah PM. Left ventricular outflow tract to left atrial communication secondary to rupture of mitral-aortic intervalvular fibrosa in infective endocarditis: diagnosis by transesophageal echocardiography and color flow imaging. J Am Coll Cardiol 1990;15:499-504.

9. Bansal RC, Moloney PM, Marsa RJ, Jacobson JG. Echocardiographic features of a mycotic aneurysm of the left ventricular outflow tract caused by perforation of mitral-aortic intervalvular fibrosa. Circulation 1983;67:930-934. 